\title{
Rotational motions in homogeneous anisotropic elastic media
}

\author{
Nguyen Dinh Pham ${ }^{1}$, Heiner Igel ${ }^{2}$, Josep de la Puente ${ }^{3}$, Martin Käser ${ }^{2}$, and \\ Michael A. Schoenberg ${ }^{4}$
}

\begin{abstract}
Rotational motions in homogeneous anisotropic elastic media are studied under the assumption of plane wave propagation. The main goal is to investigate the influences of anisotropy in the behavior of the rotational wavefield. The focus is on P-waves that theoretically do not generate rotational motion in isotropic media. By using the Kelvin-Christoffel equation, expressions are obtained of the rotational motions of body waves as a function of the propagation direction and the coefficients of the elastic modulus matrix. As a result, the amplitudes of the rotation rates and their radiation patterns are quantified and it is concluded that (1) for strong local earthquakes and typical reservoir situations quasi P-rotation rates induced by anisotropy are significant, recordable, and can be used for inverse problems; and (2) for teleseismic wavefields, anisotropic effects are unlikely to be responsible for the observed rotational energy in the P coda.
\end{abstract}

\section{INTRODUCTION}

Seismological studies are traditionally based on the observation, processing, and inversion of three orthogonal components of translational ground motions (displacement, velocity, or acceleration). In theory, to fully describe the motion of the solid volume around a point, one needs to consider not only three components of translation but also six components of strain and three components of rotations (see Aki and Richards, 2002; Stein and Wysession, 2003; Cochard et al., 2006). Although geophysical studies associated with translational motions and strain have attained a large success, the full benefits of rotational motions are still under investigation, and this type of signal has been ignored for a long time. This is mainly because sen- sors for rotational motions with sufficient resolution were not available until recently. After advances in laser technology, instruments that consistently record rotational ground motions around one axis or all three orthogonal axes have already been developed and tested (McLeod et al., 1998; Pancha et al., 2000; Schreiber et al., 2006, 2009; Wassermann et al., 2009). As a consequence, studies that aim to exploit the additional information given by collocated measurements of translational and rotational ground motion are growing rapidly (e.g., Igel et al., 2005, 2007; Cochard et al., 2006; Suryanto et al., 2006; Pham et al., 2009a, 2009b; Ferreira and Igel, 2009; Fichtner and Igel, 2009; Bernauer et al., 2009). For example, several recent studies covering many aspects of rotational ground motions have been presented in a special issue on Rotational Seismology and Engineering Applications of the Bulletin of the Seismological Society of America in May 2009 (Lee et al., 2009b). In particular, a review by Lee et al. (2009a) envisions the emergence of a new branch in the fields of observational seismology and earthquake engineering.

Once the technology for its measurement has become available, the key question is: What kind of information do we expect to retrieve from carrying out joint measurements of translations and rotations - compared with just translational observations? Early studies (Takeo and Ito, 1997) indicate that earthquake rupture histories can be better constrained with measurements of rotational motions. Furthermore, the multicomponent point measurements of rotational and translational motions allow the estimation of wavefield properties (e.g., phase velocities, propagation direction; Igel et al., 2007, Cochard et al., 2006) and allow the recovery of shear-wave structure without traveltime information (Fichtner and Igel, 2009; Bernauer et al., 2009) as well as constraining the scattering properties of the near-receiver crustal structure (Pham et al., 2009b).

An aspect that has been missing in previous studies is the role that the elastic anisotropy might play in the rotational measurements. It is well known that anisotropy is a ubiquitous phenomenon in the

Manuscript received by the Editor 16 July 2009; revised manuscript received 14 April 2010; published online 5 October 2010.

${ }^{1}$ Formerly Ludwig-Maximilians-Universität, Department of Earth and Enviornmental Sciences, Geophysics Section, Munich, Germany; presently Vietnam Academy of Science and Technology, Institute of Geophysics, Hanoi, Vietnam.

${ }^{2}$ Ludwig-Maximilians-Universität, Department of Earth and Environmental Sciences, Geophysics Section, Munich, Germany. E-mail: nguyendp@igpvast.vn; ndpseis@googlemail.com.

${ }^{3}$ Institut de Ciències del Mar, Barcelona Center for Subsurface Imaging, Department of Marine Geology, Barcelona, Spain.

${ }^{4}$ Earth Sciences Division, Lawrence Berkeley National Laboratory, Berkeley, CA (Michael A. Schoenberg passed away on 29 August 2008).

(C) 2010 Society of Exploration Geophysicists. All rights reserved. 
earth's crust and upper mantle. Many authors (e.g., Crampin, 1981, 1984; Crampin et al., 1982, 1984; Sams et al., 1993; Larner, 1993) report the influence of anisotropic effects on seismic processing and interpretation. Tsvankin (1996) emphasized that progress in seismic inversion and processing in anisotropic media depend on our ability to relate different seismic signatures to the anisotropy parameters. In reservoir and drilling engineering, the simultaneous determination of the elastic coefficients and anisotropy parameters helps in predicting flow paths for improved oil recovery, designing a hydraulic fracturing scheme, determining mud weight and selecting drill bit, and preventing hole collapse during and after drilling (Wang, 2002). At present, the anisotropy parameters can only be retrieved using laboratory measurements or arrays of standard translational seismometers deployed, at least, along a line.

At the onset of this study, there are several open questions: (1) How does anisotropy affect rotational ground motions? (2) Can rotational motions help to constrain anisotropic properties? (3) What instrument configurations are necessary to extract the relevant information? We will attempt to give preliminary answers to these questions in the following sections.

The main goal of this study is to estimate the amplitudes of the rotational motions that we can expect in various geophysical problems and their dependence on the anisotropic properties of the medium and the propagation direction. We first approach the problem in an analytical way, focusing on the solution for plane waves in linear elastic anisotropic media. Moreover, we provide estimates of the expected amplitudes of rotational motions induced by $\mathrm{P}$-waves as a function of the degree of anisotropy. Last but not least, this study confirms the fact that anisotropy alone cannot account for the P coda vertical rotational motions observed on a ring laser at the Fundamental station in Wettzell, Germany, as reported by Igel et al. (2007) and Pham et al. (2009b).

\section{FUNDAMENTAL THEORY}

In the framework of classical elasticity, a general plane-harmonicwave solution for the displacement vector $\mathbf{u}$ of body waves is

$$
\mathbf{u}=A \mathbf{n} \exp [i(\omega t-\mathbf{\kappa} \cdot \mathbf{x})]
$$

where $A$ is the ground displacement peak amplitude; $\mathbf{n}=n_{1} \mathbf{e}_{1}$ $+n_{2} \mathbf{e}_{2}+n_{3} \mathbf{e}_{3}$ is the unit vector denoting the direction of the particle displacement (i.e., the wavefield polarization; $\mathbf{e}_{i}(i=1,2,3)$ are the unit vectors along the coordinate axes); $n_{\mathrm{i}}$ $=u_{\mathrm{i}} / \sqrt{u_{1} u_{1}^{*}+u_{2} u_{2}^{*}+u_{3} u_{3}^{*}}=\left\|u_{\mathrm{i}}\right\| / A$ are the direction cosines of $\mathbf{n}$ and $i$ is the imaginary unit; superscript * represents complex conjugate; and $\|z\|$ stands for magnitude of the complex number $z$. Furthermore, the wavenumber vector is $\mathbf{\kappa}=\omega / v\left(\ell_{1} \mathbf{e}_{1}+\ell_{2} \mathbf{e}_{2}+\ell_{3} \mathbf{e}_{3}\right)$ $=\omega / v \mathbf{l}$ and the quantities $\omega, v$, and $\mathbf{l}=\ell_{1} \mathbf{e}_{1}+\ell_{2} \mathbf{e}_{2}+\ell_{3} \mathbf{e}_{3}$ are, respectively, the angular frequency, the phase velocity, and the unit vector denoting the propagation direction of the plane wave. Finally, the direction cosines of $\mathbf{l}$ are denoted $\ell_{\mathrm{i}}$.

The rotational motions $\boldsymbol{\Omega}$ generated by the plane wave expressed by equation 1 can be calculated by taking half of the curl of the displacement field (Igel et al., 2005, Cochard et al., 2006)

$$
\begin{aligned}
\mathbf{\Omega} & =\frac{1}{2}(\nabla \times \mathbf{u})=-\frac{A}{2} i \mathbf{\kappa} \times \mathbf{n} \exp [i(\omega t-\mathbf{\kappa} \cdot \mathbf{x})] \\
& =-\frac{A}{2} \frac{i \omega}{v}\left(\begin{array}{l}
\ell_{2} n_{3}-\ell_{3} n_{2} \\
\ell_{3} n_{1}-\ell_{1} n_{3} \\
\ell_{1} n_{2}-\ell_{2} n_{1}
\end{array}\right) \exp \left[i \omega\left(t-\frac{1}{v} \mathbf{l} \cdot \mathbf{x}\right)\right] .
\end{aligned}
$$

The corresponding rotation rates $\boldsymbol{\Omega}$ are then

$$
\dot{\mathbf{\Omega}}=\partial_{t} \mathbf{\Omega}=\frac{A}{2} \frac{\omega^{2}}{v}\left(\begin{array}{c}
\ell_{2} n_{3}-\ell_{3} n_{2} \\
\ell_{3} n_{1}-\ell_{1} n_{3} \\
\ell_{1} n_{2}-\ell_{2} n_{1}
\end{array}\right) \exp \left[i \omega\left(t-\frac{1}{v} \mathbf{l} \cdot \mathbf{x}\right)\right] .
$$

Looking at equation 3 , one can recognize that, in homogeneous unbounded isotropic media, $\mathrm{P}$-waves do not generate any rotational motion. This is because, for $\mathrm{P}$-waves, the vectors $\mathbf{I}$ and $\mathbf{n}$ are identical, in opposition to $\mathbf{S}$-waves, which have perpendicular $\mathbf{I}$ and $\mathbf{n}$ and hence produce rotation motion. Hence, in unbounded isotropic media, rotations can only be the consequence of an S-wave. However, this is no longer the case in anisotropic elastic media in which the phase velocity $v$ and the direction cosines $n_{\mathrm{i}}$ depend on the wave propagation direction $\ell_{\mathrm{i}}$. Hence, P-waves in anisotropic media can, in general, produce rotational motions. As a consequence, the measurement of significant rotational signals during the passage of a P-wave can be regarded as an indicator of anisotropy.

To understand the behavior of the rotational motions in anisotropic elastic media, we must first realize that the phase velocity $v$, the polarization $n_{\mathrm{i}}$, and the material properties are bound by the KelvinChristoffel equation (see e.g., Musgarve, 1970; Carcione, 2001, p. 11)

$$
\left(\boldsymbol{\Gamma}-\rho v^{2} \mathbf{I}_{3}\right) \cdot \mathbf{u}=0,
$$

where $\rho$ is the material density - a constant isotropic parameter; $\mathbf{I}_{3}$ is the $3 \times 3$ identity matrix; $\boldsymbol{\Gamma}$ is the symmetric Kelvin-Christoffel matrix

$$
\begin{aligned}
\boldsymbol{\Gamma} & =\mathbf{L} \cdot \mathbf{C} \cdot \mathbf{L}^{\mathrm{T}}, \\
\mathbf{L} & =\left(\begin{array}{ccccccc}
\ell_{1} & 0 & 0 & 0 & \ell_{3} & \ell_{2} \\
0 & \ell_{2} & 0 & \ell_{3} & 0 & \ell_{1} \\
0 & 0 & \ell_{3} & \ell_{2} & \ell_{1} & 0
\end{array}\right),
\end{aligned}
$$

and $\mathbf{C}$ is the elastic modulus matrix and is given by

$$
\mathbf{C}=\left(\begin{array}{llllll}
c_{11} & c_{12} & c_{13} & c_{14} & c_{15} & c_{16} \\
c_{12} & c_{22} & c_{23} & c_{24} & c_{25} & c_{26} \\
c_{13} & c_{23} & c_{33} & c_{34} & c_{35} & c_{36} \\
c_{14} & c_{24} & c_{34} & c_{44} & c_{45} & c_{46} \\
c_{15} & c_{25} & c_{35} & c_{45} & c_{55} & c_{56} \\
c_{16} & c_{26} & c_{36} & c_{46} & c_{56} & c_{66}
\end{array}\right) .
$$

Whenever a material possesses more than two independent parameters defining the entries of $\mathbf{C}$, the seismic wave velocities differ depending on the propagation direction and the material is called "anisotropic." A material that has all 21 coefficients in $\mathbf{C}$ independent is called a material of the triclinic symmetry class, or simply tri- 
clinic material. This is the most general case of anisotropy and includes as special cases all of the other crystalline symmetry classes (i.e., monoclinic, trigonal, tetragonal, orthorhombic, hexagonal, cubic, and isotropic). One of the most important anisotropy symmetry classes for seismological purposes is transverse isotropy. When the symmetry axis coincides with $\mathbf{e}_{3}$ the elastic modulus matrix $\mathbf{C}_{\mathrm{TI}}$ of a transversely isotropic material is (see e.g., Thomsen, 1986; Carcione, 2001, p. 6)

$$
\mathbf{C}_{\mathrm{TI}}=\left(\begin{array}{cccccc}
c_{11} & c_{12} & c_{13} & 0 & 0 & 0 \\
c_{12} & c_{11} & c_{13} & 0 & 0 & 0 \\
c_{13} & c_{13} & c_{33} & 0 & 0 & 0 \\
0 & 0 & 0 & c_{55} & 0 & 0 \\
0 & 0 & 0 & 0 & c_{55} & 0 \\
0 & 0 & 0 & 0 & 0 & c_{66}
\end{array}\right), \quad 2 c_{66}=c_{11}-c_{12} \text {. }
$$

The isotropic case can be considered as a special case of anisotropy in which $c_{11}=c_{22}=c_{33}=\lambda+2 \mu, c_{12}=c_{13}=c_{23}=\lambda, c_{44}=c_{55}$ $=c_{66}=\mu$ and all of the other coefficients are equal to zero. Here $\lambda$ and $\mu$ are the Lamé coefficients of the material.

Explicitly, the components of the Kelvin-Christoffel matrix are

$$
\begin{aligned}
\Gamma_{11}= & c_{11} \ell_{1}^{2}+c_{66} \ell_{2}^{2}+c_{55} \ell_{3}^{2}+2 c_{56} \ell_{2} \ell_{3}+2 c_{15} \ell_{3} \ell_{1} \\
& +2 c_{16} \ell_{1} \ell_{2} \\
\Gamma_{22}= & c_{66} \ell_{1}^{2}+c_{22} \ell_{2}^{2}+c_{44} \ell_{3}^{2}+2 c_{24} \ell_{2} \ell_{3}+2 c_{46} \ell_{3} \ell_{1} \\
& +2 c_{26} \ell_{1} \ell_{2} \\
\Gamma_{33}= & c_{55} \ell_{1}^{2}+c_{44} \ell_{2}^{2}+c_{33} \ell_{3}^{2}+2 c_{34} \ell_{2} \ell_{3}+2 c_{35} \ell_{3} \ell_{1} \\
& +2 c_{45} \ell_{1} \ell_{2} \\
\Gamma_{12}= & c_{16} \ell_{1}^{2}+c_{26} \ell_{2}^{2}+c_{45} \ell_{3}^{2}+\left(c_{46}+c_{25}\right) \ell_{2} \ell_{3} \\
& +\left(c_{14}+c_{56}\right) \ell_{3} \ell_{1}+\left(c_{12}+c_{66}\right) \ell_{1} \ell_{2} \\
\Gamma_{13}= & c_{15} \ell_{1}^{2}+c_{46} \ell_{2}^{2}+c_{35} \ell_{3}^{2}+\left(c_{45}+c_{36}\right) \ell_{2} \ell_{3} \\
& +\left(c_{13}+c_{55}\right) \ell_{3} \ell_{1}+\left(c_{14}+c_{56}\right) \ell_{1} \ell_{2} \\
\Gamma_{23}= & c_{56} \ell_{1}^{2}+c_{24} \ell_{2}^{2}+c_{34} \ell_{3}^{2}+\left(c_{44}+c_{23}\right) \ell_{2} \ell_{3} \\
& +\left(c_{36}+c_{45}\right) \ell_{3} \ell_{1}+\left(c_{25}+c_{46}\right) \ell_{1} \ell_{2} .
\end{aligned}
$$

It is clear that equation 4 is an eigenequation for the eigenvalues $\left(\rho v^{2}\right)_{\mathrm{m}}$ and eigenvectors $(\mathbf{u})_{\mathrm{m}}, m=1,2,3$. The dispersion relation is expressed by

$$
\operatorname{det}\left(\boldsymbol{\Gamma}-\rho v^{2} \mathbf{I}_{3}\right)=0,
$$

from which we can obtain the three phase velocities $v_{\mathrm{m}}(m=1,2,3)$ as a function of the direction cosines $\ell_{\mathrm{i}}$ and the independent coefficients of the elastic modulus matrix $\mathbf{C}$. These velocities correspond to the three body wave modes propagating in an unbounded homogeneous medium: the highest value of $v_{\mathrm{m}}$ corresponds to a quasi-Pwave (qP), whereas the other two correspond to quasi-S-waves qS1 and qS2, usually ordered in descending $v_{\mathrm{m}}$ value. For each solution $v$, we obtain from equation 4 an associated eigenvector $\mathbf{u}$; hence, the polarizations $n_{\mathrm{i}}$ are fully determined. As a consequence, finding the rotation rates produced by any plane wave is possible for a given an- isotropic material. First, we set a propagation direction and wave mode, so that we can find the values $v$ and $n_{\mathrm{i}}$ using equations 10 and 4. Substituting these values into equation 3 and setting a value for the wave's peak displacement $A$ and frequency $\omega$, one finally obtains the three components of the rotation rate $\dot{\boldsymbol{\Omega}}$.

We can now use the aforementioned process in an effort to visualize the influence that anisotropy has in the behavior of the rotational wavefield. In Figure 1, we use a plotting similar to that used by de la Puente (2008) to depict the variability of the phase velocities and peak rotation rates depending on the propagation direction. In particular, we show the values of peak rotation rates around two axes $\boldsymbol{\theta}$ and $\boldsymbol{\phi}$ generated by plane waves in a transversely isotropic (TI) medium. The three axes I (corresponding to the propagation direction), $\boldsymbol{\theta}$, and $\boldsymbol{\phi}$ of an orthogonal Cartersian coordinate system are defined in Appendix A. For the particular case of a wave propagating in the $x$ direction, we have $\mathbf{l}=\mathbf{e}_{1}, \boldsymbol{\theta}=\mathbf{e}_{3}$ and $\boldsymbol{\phi}=\mathbf{e}_{3}$, so that the three directions coincide with the Cartesian axes $x, y$, and $z$. The rotation rates in all cases are obtained by rotating the three components of the rotation rates that we obtained using equation 3 , as shown in Appendix B. We assume plane harmonic waves of peak displacement $A=10^{-5} \mathrm{~m}$ and period $T=1 \mathrm{~s}$ propagating in Mesaverde clay shale. The elastic parameters of the material are taken from Thomsen (1986) and are presented in Table 1. The plots show the highest values (phase velocity or rotation rates around $\boldsymbol{\theta}$ and $\boldsymbol{\phi}$ components) for each propagation direction. Our calculations for this particular material reveal that, for all qP-, qS1-, and qS2-waves, no rotational motion around the wave-propagation direction $\mathbf{I}$ is generated. The same result (i.e., the component of the rotation rate vector in the propagation direction $\mathbf{l}$ is zero) is also obtained for the monoclinic, orthorhombic, and tri-
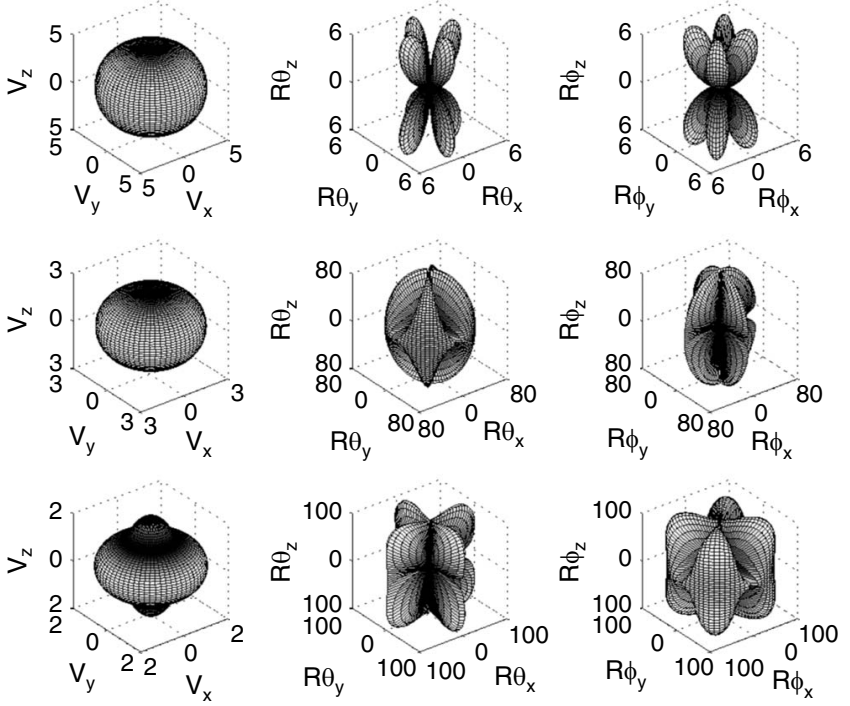

Figure 1. Example of isosurfaces of the peak phase velocity in $\mathrm{km} / \mathrm{s}$ (left column) and peak rotation rate around two axes $\boldsymbol{\theta}$ and $\boldsymbol{\phi}$ (middle and right columns) in $\mathrm{nrad} / \mathrm{s}$ as a function of the wave propagation direction. The plots shown correspond to qP- (top row), qS1(middle row), and qS2- (bottom row) waves. A plane harmonic wave of peak displacement $10^{-5} \mathrm{~m}$ and period $T=1 \mathrm{~s}$ propagating in a TI material (Mesaverde clay-shale) was assumed. The propagation direction $\mathbf{I}$ of the plane wave together with the $\boldsymbol{\theta}$ and $\boldsymbol{\phi}$ axes create an orthogonal Cartesian axis system. The vertical propagation direction in this example coincides with the symmetry axis of the TI material. For all three wave types, no rotational motion around the propagation direction $\mathbf{l}$ is generated. 
clinic materials for which the elastic coefficients were mentioned in de la Puente (2008). In fact, this result follows directly from equation 3 if we project the value of the rotation rate on the $\mathbf{I}$ direction, which results in $\mathbf{I} \cdot \dot{\mathbf{\Omega}}=0$, regardless of the material's properties.

Figure 1 shows us that the phase velocity and rotation rates strongly depend on the wave's propagation direction. The anisotropic behavior is clearly revealed by the apparition of rotational motions caused by qP-waves, which should be zero for all directions in homogeneous isotropic materials, as we mentioned previously. This again suggests that $\mathrm{qP}$ rotational motions contain information on anisotropic properties of the medium. It should be noted that there is more than one order of magnitude difference between the rotational amplitude of $\mathrm{qP}$-waves and that of $\mathrm{qS}$-waves in this example. Thus, to know if qP rotational motions can be used for inversion, it is fundamental to investigate the magnitude of the rotation rates associated with qP for different levels of anisotropy.

Although there are several symmetry classes in anisotropy, the elastic anisotropy of rocks is usually moderate and, in most cases, a TI symmetry is sufficient to describe its effects in the wavefield (e.g., Tsvankin, 1996; Guéguen and Sarout, 2009). Moreover, transverse isotropy is also acknowledged as the anisotropic case of broadest geophysical applicability (Thomsen, 1986). Thus, hereafter in this study our investigations focus only on the rotational motions caused by qP-waves in the particular case of a $\left(\mathbf{e}_{1}, \mathbf{e}_{3}\right)$ plane of a TI medium with $\mathbf{e}_{3}$ as the axis of symmetry.

Before going further, we attempt to verify the analytical equations that we have. We will compare in the following section the peak rotation rates of qP-waves obtained using this analytical solution with the results obtained from numerical simulations using the ADERDG method (the combination of a Discontinuous Galerkin finite element method and an Arbitrary high-order DERivative time integration approach developed by Dumbser and Käser, 2006 and extended to anisotropic materials by de la Puente et al., 2007).

\section{qP ROTATIONAL MOTIONS IN TI MEDIA}

When considering plane $\mathrm{P}$-waves propagating in the $\left(\mathbf{e}_{1}, \mathbf{e}_{3}\right)$ plane $\left(\ell_{2}=0, n_{2}=0\right)$ of a TI medium whose symmetry axis is $\mathbf{e}_{3}$, equation 3 can be rewritten as

$$
\dot{\mathbf{\Omega}}=\frac{A}{2} \frac{\omega^{2}}{v}\left(\begin{array}{c}
0 \\
\ell_{3} n_{1}-\ell_{1} n_{3} \\
0
\end{array}\right) \exp \left[i \omega\left(t-\frac{1}{v} \mathbf{l} \cdot \mathbf{x}\right)\right]
$$

and the Kelvin-Christoffel equation 4 can be simplified as

$$
\begin{gathered}
\left(\begin{array}{ccc}
c_{11} \ell_{1}^{2}+c_{55} \ell_{3}^{2}-\rho v^{2} & 0 & \left(c_{13}+c_{55}\right) \ell_{1} \ell_{3} \\
0 & c_{66} \ell_{1}^{2}+c_{55} \ell_{3}^{2}-\rho v^{2} & 0 \\
\left(c_{13}+c_{55}\right) \ell_{1} \ell_{3} & 0 & c_{55} \ell_{1}^{2}+c_{33} \ell_{3}^{2}-\rho v^{2}
\end{array}\right) \\
\left(\begin{array}{l}
u_{1} \\
u_{2} \\
u_{3}
\end{array}\right)=0
\end{gathered}
$$

Equation 12 shows the dispersion relation for coupled waves ( $\mathrm{qP}$ and qSV)

$$
\begin{aligned}
\left(c_{11} \ell_{1}^{2}\right. & \left.+c_{55} \ell_{3}^{2}-\rho v^{2}\right)\left(c_{55} \ell_{1}^{2}+c_{33} \ell_{3}^{2}-\rho v^{2}\right)-\left(c_{13}\right. \\
& \left.+c_{55}\right)^{2} \ell_{1}^{2} \ell_{3}^{2}=0,
\end{aligned}
$$

that allows estimating the phase velocities $v$ of qP-waves as (see e.g., Tsvankin, 1995a; Carcione, 2001)

$v=(2 \rho)^{-1 / 2} \sqrt{c_{11} \ell_{1}^{2}+c_{33} \ell_{3}^{2}+c_{55}+D}$,

$D$

$$
=\sqrt{\left[\left(c_{11}-c_{55}\right) \ell_{1}^{2}+\left(c_{55}-c_{33}\right) \ell_{3}^{2}\right]^{2}+4\left[\left(c_{13}+c_{55}\right) \ell_{1} \ell_{3}\right]^{2}} .
$$

The polarization of qP can be inferred from equations 12 and 13

$$
\frac{n_{1}}{n_{3}}=\frac{u_{1}}{u_{3}}=s \sqrt{\frac{c_{55} \ell_{1}^{2}+c_{33} \ell_{3}^{2}-\rho v^{2}}{c_{11} \ell_{1}^{2}+c_{55} \ell_{3}^{2}-\rho v^{2}}}
$$

where $s=-1$ if $\ell_{1} \ell_{3}\left(c_{11} \ell_{1}^{2}+c_{55} \ell_{3}^{2}-\rho v^{2}\right) \geq 0$; $s=1$ if $\ell_{1} \ell_{3}\left(c_{11} \ell_{1}^{2}\right.$ $\left.+c_{55} \ell_{3}^{2}-\rho v^{2}\right)<0$.

In the $\left(\mathbf{e}_{1}, \mathbf{e}_{3}\right)$ plane $u_{2}=0$, using $n_{1}^{2}+n_{3}^{2}=1$ and equation 15 we obtain

$$
\begin{aligned}
& n_{1}=s \sqrt{\frac{c_{55} \ell_{1}^{2}+c_{33} \ell_{3}^{2}-\rho v^{2}}{c_{11} \ell_{1}^{2}+c_{33} \ell_{3}^{2}+c_{55}-2 \rho v^{2}}}, \\
& n_{3}=\sqrt{\frac{c_{11} \ell_{1}^{2}+c_{55} \ell_{3}^{2}-\rho v^{2}}{c_{11} \ell_{1}^{2}+c_{33} \ell_{3}^{2}+c_{55}-2 \rho v^{2}}} .
\end{aligned}
$$

Substituting equations $14 \mathrm{a}, 16$, and 17 into equation 11 , the rotation rates induced by plane $\mathrm{P}$-waves propagating in the $\left(\mathbf{e}_{1}, \mathbf{e}_{3}\right)$ plane of a TI material with $\mathbf{e}_{3}$ as the axis of symmetry can be expressed as a function of the propagation direction as

$$
\begin{aligned}
\dot{\mathbf{\Omega}}= & A \omega^{2} \sqrt{\frac{\rho}{2\left(c_{11} \ell_{1}^{2}+c_{33} \ell_{3}^{2}+c_{55}+D\right)}}\left(\begin{array}{c}
0 \\
\ell_{3} n_{1}-\ell_{1} n_{3} \\
0
\end{array}\right) \\
& \times \exp \left[i \omega\left(t-\frac{1}{v} \mathbf{l} \cdot \mathbf{x}\right)\right] \\
n_{1}= & s \sqrt{\frac{D-\left(c_{55}-c_{11}\right) \ell_{1}^{2}-\left(c_{33}-c_{55}\right) \ell_{3}^{2}}{2 D}} \\
n_{3}= & \sqrt{\frac{D+\left(c_{55}-c_{11}\right) \ell_{1}^{2}+\left(c_{33}-c_{55}\right) \ell_{3}^{2}}{2 D}} \\
s= & -1 \quad \text { if } \quad \ell_{1} \ell_{3}\left[\left(c_{11}-c_{55}\right) \ell_{1}^{2}+\left(c_{55}-c_{33}\right) \ell_{3}^{2}-D\right] \\
\geq & 0,
\end{aligned}
$$

Table 1. Elastic coefficients for the TI material (Mesaverde clay shale), given in $\left[10^{9} \mathrm{~N} \cdot \mathrm{m}^{-2}\right]$.

\begin{tabular}{llllllllll}
$\rho$ & $c_{11}$ & $c_{12}$ & $c_{13}$ & $c_{22}$ & $c_{23}$ & $c_{33}$ & $c_{44}$ & $c_{55}$ & $c_{66}$ \\
\hline 2590 & 66.6 & 19.7 & 39.4 & 66.6 & 39.4 & 39.9 & 10.9 & 10.9 & 23.45 \\
\hline
\end{tabular}

The material density $\rho$ is given in $\left[\mathrm{kg} \cdot \mathrm{m}^{-3}\right]$. All of the other coefficients are zero. 


$$
s=1 \quad \text { if } \quad \ell_{1} \ell_{3}\left[\left(c_{11}-c_{55}\right) \ell_{1}^{2}+\left(c_{55}-c_{33}\right) \ell_{3}^{2}-D\right]<0 .
$$

Assuming that $\psi$ is the angle between the wave propagation direction $\mathbf{I}$ and the symmetry axis $\mathbf{e}_{3}$ of the TI medium, we have

$$
\begin{aligned}
& \ell_{1}=\sin \psi \\
& \ell_{3}=\cos \psi .
\end{aligned}
$$

This substitution allows us to calculate the rotation rates induced by plane $\mathrm{P}$-waves propagating in the $\left(\mathbf{e}_{1}, \mathbf{e}_{3}\right)$ plane of a TI material as a function of $\psi$.

To evaluate the correctness of the analytical solution, we model the synthetic seismograms created by a plane P-wave of dominant period of $1 \mathrm{~s}$ propagating upward through a TI medium that the symmetry axis belongs to the $\left(\mathbf{e}_{1}, \mathbf{e}_{3}\right)$ plane. A model of $30,000 \mathrm{~m}$ length, $30,000 \mathrm{~m}$ width, and 20,000 $\mathrm{m}$ depth is used. The material chosen is Mesaverde clay shale (Table 1). To check the effect of different incidence angles, we rotate the symmetry axis of the material a full circle inside the $\left(\mathbf{e}_{1}, \mathbf{e}_{3}\right)$ plane with steps of $5^{\circ}$. Such rotation of the material, having fixed the propagation direction of the plane wave, is equivalent to a test in which the material is fixed and the wave propagation direction $I$ is rotated. In total, we investigate 72 different cases characterized by the angle between the material's symmetry axis and the vertical axis, ranging from $0^{\circ}$ to $360^{\circ}$.

Six-component seismograms (three components of rotation rates and three components of translational velocities) are calculated using the ADER-DG method. The modeling parameters are detailed in Table 2 .

The simulation results show that there is only rotational signal around the $\mathbf{e}_{2}$ axis, as we expected from theory (see equation 18). We superimpose the normalized peak rotation rates around the $\mathbf{e}_{2}$ axis obtained from the simulations and the analytic approach as a function of the angle $\psi$ between the wave propagation direction and the symmetry axis of the material in a polar coordinate system. The excellent fit between both results presented in Figure 2 indicates the consistency of the analytical and numerical solutions.

We summarize that, for homogeneous anisotropic elastic media, we can estimate the rotational motions caused by a certain plane wave as a function of propagation direction and elastic coefficients. The anisotropic behavior is clearly revealed by the appearance of $\mathrm{qP}$ rotational motions. In the following, we extend the analytical equa-

Table 2. The modeling parameters used in this study.

Mesh type

Element edge length

Total number of elements

Polynomial degree inside elements

Number of processors

Length of seismograms

Boundary conditions

Average time step

Run time per simulation tions to be able to infer the magnitude of the rotation rates associated with qP depending on the degree of anisotropy of the material.

\section{MAGNITUDE OF qP ROTATIONS IN TERMS OF THOMSEN PARAMETERS}

As reported by Thomsen (1986), for the case of transverse isotropy, an alternative and more descriptive set of parameters can be used to fully describe the medium's properties in substitution of the five elastic coefficients presented in equation 8 . These parameters are the two elastic equivalent quantities $\alpha_{0}$ and $\beta_{0}$ and three coefficients $\gamma$, $\varepsilon$, and $\delta *$ (usually called Thomsen parameters) expressed as follows

$$
\begin{gathered}
\alpha_{0}=\sqrt{\frac{c_{33}}{\rho}}, \\
\beta_{0}=\sqrt{\frac{c_{55}}{\rho}}, \\
\varepsilon=\frac{c_{11}-c_{33}}{2 c_{33}}, \\
\gamma=\frac{c_{66}-c_{55}}{2 c_{55}},
\end{gathered}
$$

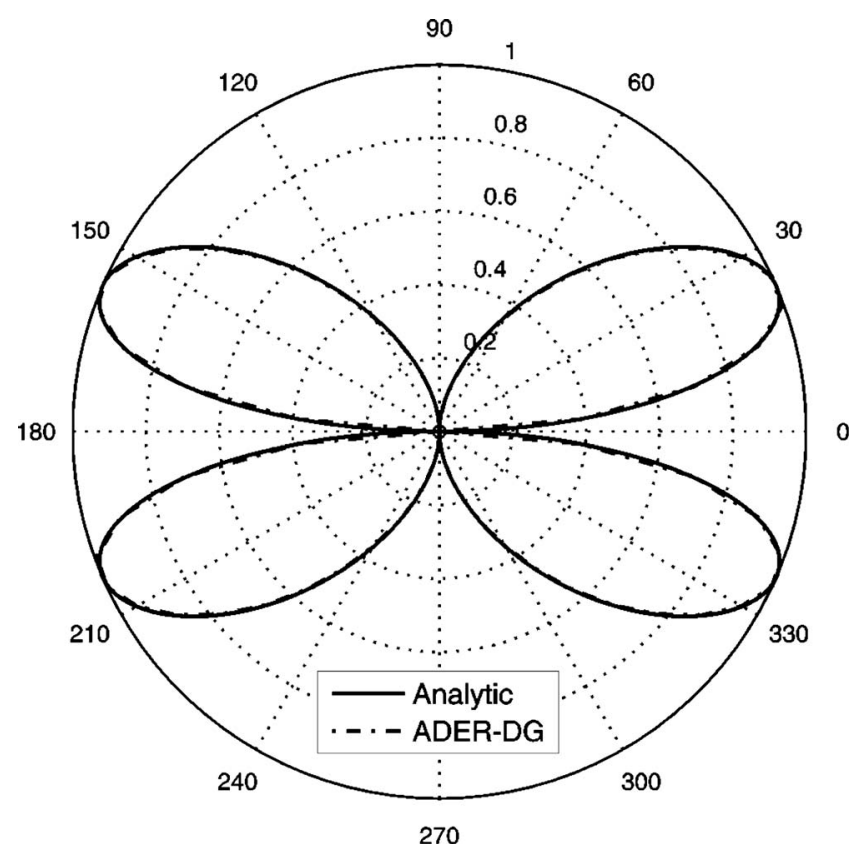

Figure 2. Variations of the normalized peak rotation rate induced by a qP-wave of period $T=1 \mathrm{~s}$ propagating in a vertical plane of a TI medium (Mesaverde clay shale) as a function of the angle $\psi$ between the wave propagation direction and the symmetry axis of the medium. The results are presented in a polar coordinate system. Solid line $=$ analytic results, dash-dot line $=$ numerical simulation results using the ADER-DG method. 


$$
\delta^{*}=\frac{1}{2 c_{33}^{2}}\left[2\left(c_{13}+c_{55}\right)^{2}-\left(c_{33}-c_{55}\right)\left(c_{11}+c_{33}-2 c_{55}\right)\right] .
$$

The coefficients $\gamma, \varepsilon$, and $\delta^{*}$ are dimensionless anisotropy parameters that fully characterize the strength of the anisotropy. Thus, to quantify the magnitude of $\mathrm{qP}$ rotations in anisotropic elastic media, in this section we investigate the dependence of the peak rotation rate values of qP-waves on these Thomsen parameters.

As mentioned by Tsvankin and Thomsen, 1994 and Tsvankin, 1995b, the parameter $\gamma$ does not affect the propagation of the qP- or the qSV-waves in TI media. Only the SH mode depends on this parameter. This can also be recognized from equation 13. For the coupled waves, neither the phase velocities nor the Kelvin-Christoffel equations depend on $c_{66}$. As a consequence, the rotation rates induced by qP-waves are also independent of $c_{66}$ (see equation 18). This means that, in a certain TI medium with a constant value of $\beta_{0}$ (or equivalently $c_{55}$ ), the qP rotation rates are independent of $\gamma$ (see equations 21 and 23). Hence, we consequently skip the parameter $\gamma$ in the following. We quantify the peak rotation rates of the qP-waves generated in TI materials with different levels of anisotropy only as a function of the two parameters $\varepsilon$ and $\delta^{*}$.

We define a set of elastic modulus matrices $\mathbf{C}$ by assuming fixed values of $\alpha_{0}, \beta_{0}, \rho$, and $\gamma$ and letting $\varepsilon$ and $\delta^{*}$ take different values. For each $\left(\varepsilon, \delta^{*}\right)$ couple, we aim to obtain the peak rotation rates produced by a qP-wave traveling in any direction. To this goal, for each elastic modulus matrix $\mathbf{C}$ and for each propagation direction, we first extract the phase velocity and the polarization of the considered qPwave by solving equation 10 . The corresponding rotation rates are calculated using equation 3 . The corresponding peak rotation rate can be then obtained trivially from the full rotation rate vector. In this way, we obtain the peak qP rotation rate that can be generated by a certain plane wave in the considered materials, each characterized by its $\varepsilon$ and $\delta^{*}$ values, in all propagation directions $\mathbf{l}$. We consider values of $\varepsilon$ ranging from 0 to 0.35 and those of $\delta^{*}$ from -0.3 to 1 . These values cover most TI cases observed (Thomsen, 1986). Four case studies are investigated: (1) a plane wave of peak ground displacement (PGD) $355.78 \mathrm{~nm}$ and period $T=1 \mathrm{~s}$ (caused by a distant earthquake) propagating through bedrock; (2) a plane wave of PGD $=48 \times 10^{-4} \mathrm{~m}$ and period $T=0.8 \mathrm{~s}$ (can be caused by a local earthquake M7.0, epicenter distance $100 \mathrm{~km}$ ) propagating through bedrock; (3) a plane wave of PGD $=10^{-6} \mathrm{~m}$ and frequency $f=150 \mathrm{~Hz}$ (can originate from a microseismic event of $\mathrm{M}_{\mathrm{w}}=0$ at hypocenter distance of approximately $1 \mathrm{~km}$ ) propagating through reservoir rock; and (4) a plane wave of $\mathrm{PGD}=180.35 \times 10^{-6} \mathrm{~m}$ and $f$ $=5 \mathrm{~Hz}$ (can be caused by an air-gun experiment) propagating though reservoir rock (see Chen et al., 2008). For each case study, reasonable values of $\alpha_{0}, \beta_{0}$, and mass density $\rho$ are used and are detailed in Table 3. Because of the independence of the qP rotation rates on $\gamma$, any arbitrary value of this Thomsen parameter can be taken. Here we note that the first case study is based on measurements of the 25 September 2003 M8.1 Tokachi-oki earthquake at the Wettzell station in southeast Germany (see Pham et al., 2009a) except for the anisotropy, which has been solely added for the purpose of the research presented here.

We compute for each of the four study cases the peak rotation rates of qP-waves generated in different TI materials for a set of $\varepsilon$ and $\delta *$ values. The results are given in Figures 3-6. Every point in the plot depicts the maximum $\mathrm{qP}$ rotation rate among all propagation directions for a given couple of values of $\left(\varepsilon, \delta^{*}\right)$. A general trend that can be observed is that peak qP rotation rates are higher with increasing values of $\varepsilon$ and $\delta^{*}$. In particular, if we look at the results obtained for the case of the distant earthquake (Figure 3), we can safely conclude that, if we consider weakly anisotropic bedrock under Wettzell (Lee et al., 2009b), we should measure qP rotation rates of at most 5 $\times 10^{-11} \mathrm{rad} / \mathrm{s}$. This value is much smaller than the amplitude $(6.3$ $\times 10^{-10} \mathrm{rad} / \mathrm{s}$ ) observed in $\mathrm{P}$ coda rotations, as reported by Pham et al. (2009a). However, for the other three study cases, significant amplitudes of qP rotation rates are expected (Figures 4-6). With peak $\mathrm{qP}$ rotation rates in the order of $\mu \mathrm{rad} / \mathrm{s}$, even in weakly anisotropic materials the signals can be recorded with current instrument technology (Nigbor et al., 2009; Wassermann et al., 2009).

\section{DISCUSSION}

The rotational motion of $\mathrm{qP}$-waves studied in this paper is another expression of the polarization deviation of the $\mathrm{P}$-waves in anisotropic media studied in earlier papers (e.g., Pšenčík and Gajewski, 1998; Zheng and Pšenčík, 2002). However, at the beginning of this study it was not clear how anisotropy affects rotational ground motions and if rotational motions can help putting constraints on anisotropic properties. The results obtained in this paper under the assumption of plane wave propagation show that (1) anisotropic behavior in elastic media can be recognized from rotational motion records, especially by the appearance of qP rotations; (2) for typical reservoir situations and strong local earthquakes qP rotation signals are significant, recordable, and can be used for inverse problems.

It should be noted that our original motivation to study the relation between anisotropy and rotational motions comes from efforts to explain the observed P coda rotations reported by Igel et al. (2007) and Pham et al. (2009b). At the beginning of this study, it was unclear whether rotational motions induced by qP-waves in anisotropic media contribute to the observed rotational signals in the P coda. Relat-

Table 3. Parameters used for quantifying peak rotation rates of qP-waves in terms of the Thomsen parameters for four case studies.

\begin{tabular}{lccccc} 
Case study & $\begin{array}{c}\text { Peak ground } \\
\text { displacement }(\mathrm{m})\end{array}$ & $\begin{array}{c}\text { Considered } \\
\text { period }(\mathrm{s})\end{array}$ & $\alpha_{0}(\mathrm{~m} / \mathrm{s})$ & $\beta_{0}(\mathrm{~m} / \mathrm{s})$ & $\rho\left(\mathrm{kg} / \mathrm{m}^{3}\right)$ \\
\hline 1. Bedrock/distant earthquake & $355.78 \times 10^{-9}$ & 1 & 6600 & 3700 & 2900 \\
2. Bedrock/local earthquake & $48 \times 10^{-4}$ & 0.8 & 6600 & 3700 & 2900 \\
3. Reservoir/microseismic event & $10^{-6}$ & $1 / 150$ & 3928 & 2055 & 2590 \\
4. Reservoir/air-gun experiment & $180.35 \times 10^{-6}$ & 0.2 & 3928 & 2055
\end{tabular}




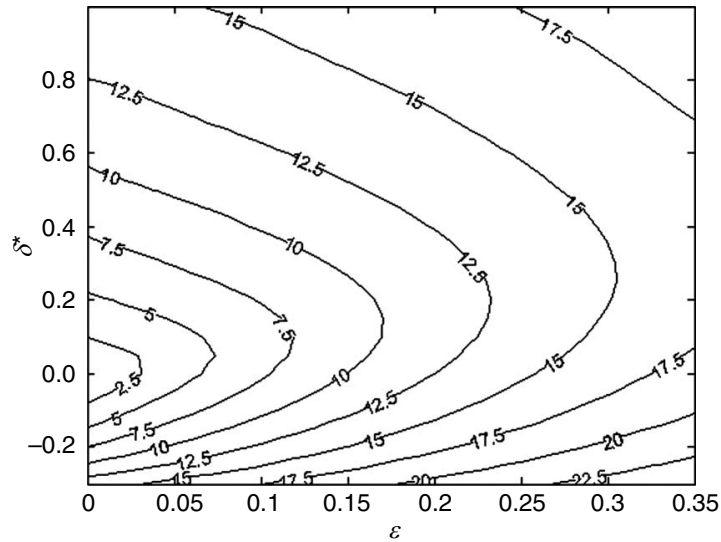

Figure 3. The maximum peak rotation rates (in $10^{-11} \mathrm{rad} / \mathrm{s}$ ) caused by plane P-waves in a full space TI medium as a function of the Thomsen parameters $\varepsilon$ and $\delta *$, as expected for a distant earthquake with PGD of $355.78 \mathrm{~nm}$ and period $T=1 \mathrm{~s}$. A vertical P velocity $\alpha_{0}$ $=6600 \mathrm{~m} / \mathrm{s}$, vertical S velocity $\beta_{0}=3700 \mathrm{~m} / \mathrm{s}$, and $\rho=2900$ $\mathrm{kg} / \mathrm{m}^{3}$ were assumed. Every point in the plot depicts the maximum $\mathrm{qP}$ rotation rate among all propagation directions for a given couple of values of $\left(\varepsilon, \delta^{*}\right)$.

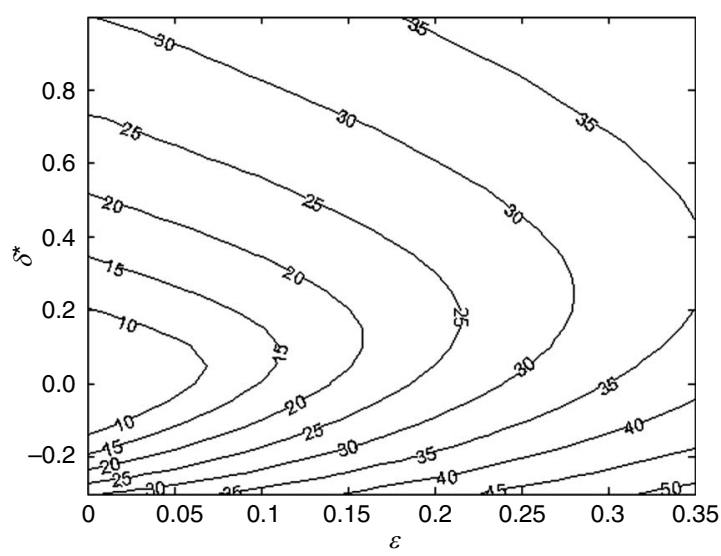

Figure 4. The maximum peak rotation rate (in $10^{-7} \mathrm{rad} / \mathrm{s}$ ) caused by qP-waves in a full space TI medium as a function of the Thomsen parameters $\varepsilon$ and $\delta^{*}$, as expected for a local earthquake with PGD 48 $\times 10^{-4} \mathrm{~m}$ and period $T=0.8 \mathrm{~s}$. A vertical $\mathrm{P}$ velocity $\alpha_{0}$ $=6600 \mathrm{~m} / \mathrm{s}$, vertical S velocity $\beta_{0}=3700 \mathrm{~m} / \mathrm{s}$, and $\rho=2900$ $\mathrm{kg} / \mathrm{m}^{3}$ were assumed. Every point in the plot depicts the maximum $\mathrm{qP}$ rotation rate among all propagation directions for a given couple of values of $\left(\varepsilon, \delta^{*}\right)$.

ed to this, several results obtained in the study presented here need to be mentioned. First, although anisotropy does generate qP rotations, for teleseismic wavefields the rotational energy induced by anisotropic effects is expected to be much smaller than the observed one in the P coda. Second, it is evident from equation 3 that translational acceleration and the resulting $\mathrm{qP}$ rotation rates would have the same waveforms coinciding in time. However, as reported by Pham et al. (2009b), the P coda rotations observed at the Wettzell station come later than the appearance of direct P-waves. These results demonstrate that anisotropic $\mathrm{qP}$ rotational signals cannot be the main cause of the observed $\mathrm{P}$ coda rotations. The difference in the arrival time of anisotropic $\mathrm{qP}$ rotational motions and scattering $\mathrm{P}$ coda rotations can be used to separate these two cases for specific purposes when a heterogeneous medium is present.

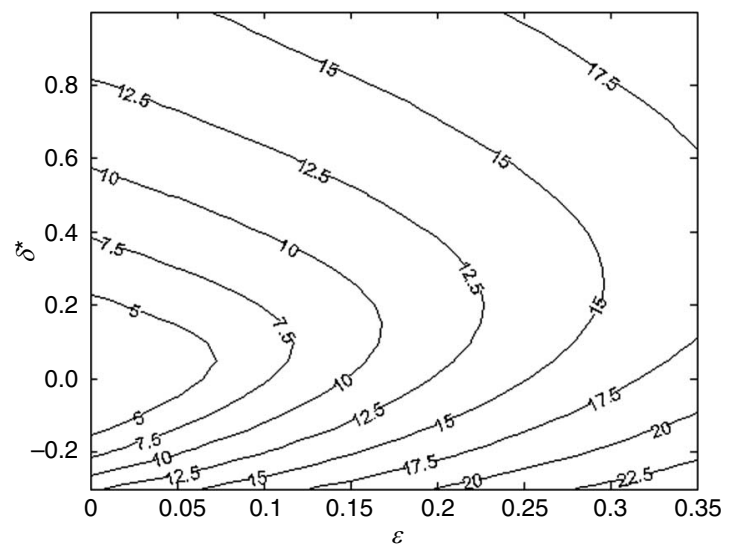

Figure 5. The maximum peak rotation rate (in $10^{-6} \mathrm{rad} / \mathrm{s}$ ) caused by $\mathrm{qP}$-waves in a full space TI medium as a function of the Thomsen parameters $\varepsilon$ and $\delta^{*}$ as expected for a reservoir microseismic event with PGD of $10^{-6} \mathrm{~m}$ and frequency $f=150 \mathrm{~Hz}$. A vertical P velocity $\alpha_{0}=3928 \mathrm{~m} / \mathrm{s}$, vertical S velocity $\beta_{0}=2055 \mathrm{~m} / \mathrm{s}$, and $\rho$ $=2590 \mathrm{~kg} / \mathrm{m}^{3}$ were assumed. Every point in the plot depicts the maximum $\mathrm{qP}$ rotation rate among all propagation directions for a given couple of values of $\left(\varepsilon, \delta^{*}\right)$.

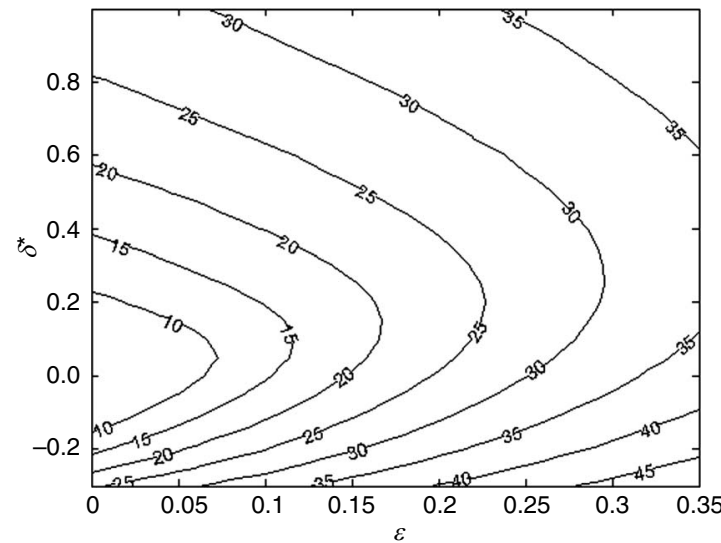

Figure 6. The maximum peak rotation rate (in $10^{-7} \mathrm{rad} / \mathrm{s}$ ) caused by $\mathrm{qP}$-waves in a full space TI medium as a function of the Thomsen parameters $\varepsilon$ and $\delta^{*}$ as expected for an air-gun experiment in a reservoir area with PGD $180.35 \times 10^{-6} \mathrm{~m}$ and frequency $f=5 \mathrm{~Hz}$. A vertical $\mathrm{P}$ velocity $\alpha_{0}=3928 \mathrm{~m} / \mathrm{s}$, vertical $\mathrm{S}$ velocity $\beta_{0}$ $=2055 \mathrm{~m} / \mathrm{s}$, and $\rho=2590 \mathrm{~kg} / \mathrm{m}^{3}$ were assumed. Every point in the plot depicts the maximum qP rotation rate among all propagation directions for a given couple of values of $\left(\varepsilon, \delta^{*}\right)$.

\section{CONCLUSIONS}

In summary, we conclude that rotational motions contain additional information about the material's anisotropic properties and that joint measurements of translational and rotational motions of qP-waves in anisotropic media might allow one to constrain anisotropic parameters. Our results not only demonstrate the potential benefit of measurements of rotational ground motions but also pose several issues that need to be addressed in the future.

1) Because we have shown that the amplitudes of the qP rotational motions generated in anisotropic materials are high enough to be observed and recorded, one of the key questions is: Can we extract additional information about the material's properties using $\mathrm{qP}$ rotations? 
2) In the study presented here, we focus mainly on qP-waves, the rotational motions of which are completely independent of the Thomsen parameter $\gamma$. Thus, the consideration of this measure of $\mathrm{S}$ anisotropy (i.e., $\gamma$ ) is out of the framework of this paper. Theoretically, qS motions also contain additional information. Can we constrain the Thomsen parameter $\gamma$ using measurements of rotational motions of qS-waves? What additional information can be extracted if these motions are used?

3) The well-known result for isotropic media that the rotation vector is orthogonal to the propagation direction (i.e., the component of the rotation rate vector in the propagation direction is zero) is theoretically and numerically demonstrated to also hold for anisotropic media. This opens an opportunity to investigate scattering properties of heterogeneous media on the basis of variations of rotational motions around the wave propagation direction.

Finally, the applicability of the presented theory to real data will rest on the ability to develop reliable sensors (borehole- and surfacebased) for rotational motions with a broad enough frequency bandwidth and dynamic range.

\section{ACKNOWLEDGMENTS}

This research was supported by the Geophysics Section of Ludwig-Maximilians-Universität Munich, the Vietnamese government (project 322 and Vietnam's National Foundation for Science and Technology Development), and the German Academic Exchange Service (DAAD). We thank the KONWIHR project and the Munich Leibniz Supercomputing Centre for computational resources and the European Human Resources Mobility Program (SPICE Project). We thank Mirko van der Baan and four anonymous reviewers for their suggestions and comments that helped to improve the manuscript. J. de la Puente acknowledges the funding of the Kaleidoscope project, supported by Repsol-YPF. H. Igel thanks Mike Schoenberg, who passed away in 2008, for his early thoughts on the problem, the discussions they had a few days before his death, and above all for his friendship and support.

\section{APPENDIX A \\ ORTHOGONAL CARTESIAN COORDINATE SYSTEM $\ell, \Theta, \Phi$}

For the purpose of visualization of the influence that anisotropy has in the behavior of the rotational wavefield, an orthogonal Cartesian coordinate system I, $\boldsymbol{\theta}, \boldsymbol{\phi}$ is used in the paper (see Figure A-1). The system is defined as follows. We first build the unit vector $\mathbf{I}$ $=\left(\ell_{1}, \ell_{2}, \ell_{3}\right)$ so that its axis coincides with the wave propagation direction. Furthermore, we generate a set of three auxiliary vectors $\mathbf{p}$, $\mathbf{q}$, and $\mathbf{r}$ that will help us define the two remaining axes of our Cartesian coordinate system. These are defined as

$$
\begin{aligned}
& \mathbf{p}=\left(\ell_{1},-\ell_{2}, 0\right), \\
& \mathbf{q}=\left(0,-\ell_{3}, \ell_{2}\right), \\
& \mathbf{r}=\left(\ell_{3}, 0,-\ell_{1}\right) .
\end{aligned}
$$

The definition of the two remaining Cartesian axes will depend on whether the auxiliary vectors are collinear or not and whether one of them is the vector $\mathbf{0}$. The three distinct cases are described as follows. If $|\mathbf{I} \times \mathbf{p}|>0$, then $\boldsymbol{\theta}$ is defined as

$$
\boldsymbol{\theta}=\mathbf{l} \times \mathbf{p}=\left(\ell_{2} \ell_{3}, \ell_{1} \ell_{3},-2 \ell_{1} \ell_{2}\right) .
$$

If $|\mathbf{I} \times \mathbf{p}|=0$ and $|\mathbf{I} \times \mathbf{q}|>0$, then $\boldsymbol{\theta}$ is defined as

$$
\boldsymbol{\theta}=\mathbf{l} \times \mathbf{q}=\left(\ell_{2}^{2}+\ell_{3}^{2},-\ell_{1} \ell_{2},-\ell_{1} \ell_{3}\right) .
$$

If $|\mathbf{I} \times \mathbf{p}|=0$ and $|\mathbf{I} \times \mathbf{q}|=0$, then $\boldsymbol{\theta}$ is defined as

$$
\boldsymbol{\theta}=\mathbf{l} \times \mathbf{r}=\left(-\ell_{1} \ell_{2}, \ell_{3}^{2}+\ell_{1}^{2},-\ell_{2} \ell_{3}\right) .
$$

The vector $\boldsymbol{\phi}$ is always obtained by cross product of $\mathbf{l}$ and $\boldsymbol{\theta}$,

$$
\boldsymbol{\phi}=\mathbf{I} \times \boldsymbol{\theta} .
$$

\section{APPENDIX B}

\section{AXIS TRANSFORMATIONS OF ROTATIONS}

To set up the relationship between components of a rotational motion in two orthogonal Cartesian coordinate systems $(x, y, z)$ and $\left(x^{*}, y^{*}, z^{*}\right)$, we start by recalling the definition of the rotation rate

$$
\left(\begin{array}{c}
\dot{\Omega}_{\mathrm{x}} \\
\dot{\Omega}_{\mathrm{y}} \\
\dot{\Omega}_{\mathrm{z}}
\end{array}\right)=\frac{1}{2} \nabla \times \mathbf{V}=\frac{1}{2}\left(\begin{array}{c}
\partial_{\mathrm{y}} V_{\mathrm{z}}-\partial_{\mathrm{z}} V_{\mathrm{y}} \\
\partial_{\mathrm{z}} V_{\mathrm{x}}-\partial_{\mathrm{x}} V_{\mathrm{z}} \\
\partial_{\mathrm{x}} V_{\mathrm{y}}-\partial_{\mathrm{y}} V_{\mathrm{x}}
\end{array}\right),
$$

where $\mathbf{V}$ is the ground translational velocity and $\dot{\Omega}$ is the rotation rate.

In the $\left(x^{*}, y^{*}, z^{*}\right)$ system, we can similarly find that

$$
\dot{\Omega}_{\mathrm{x}}^{*}=\frac{1}{2}\left(\partial_{\mathrm{y} *} V_{\mathrm{z}}^{*}-\partial_{\mathrm{z}} V_{\mathrm{y}}^{*}\right) .
$$

The relationship between the components of the translational motion in the two systems can be expressed as

$$
\mathbf{V}^{*}=A \mathbf{V},
$$

which explicitly can be written as

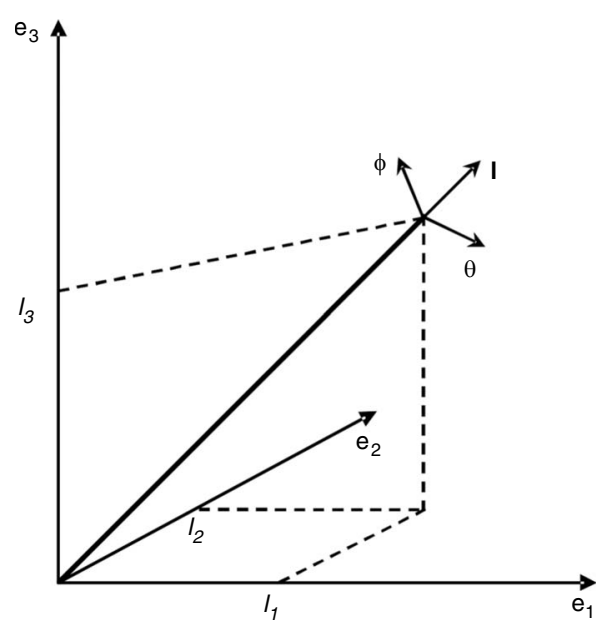

Figure A-1. Illustration of the orthogonal Cartersian coordinate system $\mathbf{I}, \boldsymbol{\theta}, \boldsymbol{\phi}$ used in this study. 


$$
\left(\begin{array}{c}
V_{\mathrm{x}}^{*} \\
V_{\mathrm{y}}^{*} \\
V_{\mathrm{z}}^{*}
\end{array}\right)=\left(\begin{array}{lll}
a_{11} & a_{12} & a_{13} \\
a_{21} & a_{22} & a_{23} \\
a_{31} & a_{32} & a_{33}
\end{array}\right)\left(\begin{array}{c}
V_{\mathrm{x}} \\
V_{\mathrm{y}} \\
V_{\mathrm{z}}
\end{array}\right),
$$

where $a_{\mathrm{ij}}=\mathbf{e}_{\mathrm{i}}{ }^{*} \cdot \mathbf{e}_{\mathrm{j}}=\cos \alpha_{\mathrm{ij}}(i=1,2,3 ; j=1,2,3)$ are the so-called direction cosines; $\alpha_{\mathrm{ij}}$ are angles between two sets of axes; and $\left(\mathbf{e}_{1}{ }^{*}, \mathbf{e}_{2}{ }^{*}, \mathbf{e}_{3}{ }^{*}\right)$ and $\left(\mathbf{e}_{1}, \mathbf{e}_{2}, \mathbf{e}_{3}\right)$ denote the unit basis vectors of the axes of the two systems.

Using B-4, equation B-2 can be rewritten as

$$
\begin{aligned}
\dot{\boldsymbol{\Omega}}_{\mathrm{x}}^{*}= & \frac{1}{2}\left[\left(a_{31} \partial_{\mathrm{y} *} V_{\mathrm{x}}+a_{32} \partial_{\mathrm{y} *} V_{\mathrm{y}}+a_{33} \partial_{\mathrm{y} *} V_{\mathrm{z}}\right)-\left(a_{21} \partial_{\mathrm{z}} V_{\mathrm{x}}\right.\right. \\
& \left.\left.+a_{22} \partial_{\mathrm{z}} V_{\mathrm{y}}+a_{23} \partial_{\mathrm{z} *} V_{\mathrm{z}}\right)\right] .
\end{aligned}
$$

Now we use the Jacobian of the transformation to obtain the derivatives in the new coordinate system.

$$
\partial_{x^{*}}=\frac{\partial x}{\partial x^{*}} \partial_{x}+\frac{\partial y}{\partial x^{*}} \partial_{\mathrm{y}}+\frac{\partial z}{\partial x^{*}} \partial_{\mathrm{z}}=a_{11} \partial_{\mathrm{x}}+a_{12} \partial_{\mathrm{y}}+a_{13} \partial_{\mathrm{z}}
$$

$$
\partial_{\mathrm{y}^{*}}=\frac{\partial x}{\partial y^{*}} \partial_{\mathrm{x}}+\frac{\partial y}{\partial y^{*}} \partial_{\mathrm{y}}+\frac{\partial z}{\partial y^{*}} \partial_{\mathrm{z}}=a_{21} \partial_{\mathrm{x}}+a_{22} \partial_{\mathrm{y}}+a_{23} \partial_{\mathrm{z}},
$$

$$
\partial_{z^{*}}=\frac{\partial x}{\partial z^{*}} \partial_{x}+\frac{\partial y}{\partial z^{*}} \partial_{y}+\frac{\partial z}{\partial z^{*}} \partial_{z}=a_{31} \partial_{x}+a_{32} \partial_{y}+a_{33} \partial_{z} .
$$

Substituting B-6-B-8 and B-5, we find that

$$
\begin{aligned}
\dot{\Omega}_{\mathrm{x}}^{*}= & \frac{1}{2}\left[\left(a_{33} a_{22}-a_{32} a_{23}\right)\left(\frac{\partial V_{\mathrm{z}}}{\partial y}-\frac{\partial V_{\mathrm{y}}}{\partial z}\right)+\left(a_{31} a_{23}\right.\right. \\
& \left.-a_{33} a_{21}\right)\left(\frac{\partial V_{\mathrm{x}}}{\partial z}-\frac{\partial V_{\mathrm{z}}}{\partial x}\right)+\left(a_{32} a_{21}-a_{31} a_{22}\right)\left(\frac{\partial V_{\mathrm{y}}}{\partial x}\right. \\
& \left.\left.-\frac{\partial V_{\mathrm{x}}}{\partial y}\right)\right]
\end{aligned}
$$

which leads to the final form

$$
\begin{aligned}
\dot{\Omega}_{\mathrm{x}}{ }^{*}= & \left(a_{33} a_{22}-a_{32} a_{23}\right) \dot{\Omega}_{\mathrm{x}}+\left(a_{31} a_{23}-a_{33} a_{21}\right) \dot{\Omega}_{\mathrm{y}}+\left(a_{32} a_{21}\right. \\
& \left.-a_{31} a_{22}\right) \dot{\Omega}_{\mathrm{z}}
\end{aligned}
$$

Similarly, we also obtain

$$
\begin{aligned}
\dot{\Omega}_{\mathrm{y}}{ }^{*}= & \left(a_{13} a_{32}-a_{33} a_{12}\right) \dot{\Omega}_{\mathrm{x}}+\left(a_{11} a_{33}-a_{31} a_{13}\right) \dot{\Omega}_{\mathrm{y}}+\left(a_{12} a_{31}\right. \\
& \left.-a_{32} a_{11}\right) \dot{\Omega}_{\mathrm{z}}, \\
\dot{\Omega}_{\mathrm{z}}{ }^{*}= & \left(a_{23} a_{12}-a_{13} a_{22}\right) \dot{\Omega}_{\mathrm{x}}+\left(a_{21} a_{13}-a_{11} a_{23}\right) \dot{\Omega}_{\mathrm{y}}+\left(a_{11} a_{22}\right. \\
& \left.-a_{12} a_{21}\right) \dot{\Omega}_{\mathrm{z}} .
\end{aligned}
$$

The last three equations can be written in the compact form

$$
\dot{\mathbf{\Omega}}^{*}=\left(\mathrm{A}^{-1}\right)^{\mathrm{T}} \dot{\mathbf{\Omega}}
$$

which we can compare to B-3 to see how the rotation-rate vector can be transformed into a rotated Cartesian system once we know the expression of the unitary vectors $\left(\mathbf{e}_{1}{ }^{*}, \mathbf{e}_{2}{ }^{*}, \mathbf{e}_{3}{ }^{*}\right)$ that form the basis of the new system.

\section{REFERENCES}

Aki, K., and P. G. Richards, 2002, Quantitative seismology, 2nd ed.: University Science Books.

Bernauer, M., A. Fichtner, and H. Igel, 2009, Inferring earth structure from combined measurements of rotational and translational ground motions: Geophysics, 74, no. 6, WCD41-WCD47.

Carcione, J. M., 2001, Wave fields in real media: wave propagation in anisotropic, anelastic and porous media, 31: Pergamon.

Chen, Y., L. Liu, H. Ge, B. Liu, and X. Qiu, 2008, Using an airgun array in a land reservoir as the seismic source for seismotectonic studies in northern China: experiments and preliminary results: Geophysical Prospecting, 56, no. 4, 601-612.

Cochard, A., H. Igel, B. Schuberth, W. Suryanto, A. Velikoseltsev, U. Schreiber, J. Wassermann, F. Scherbaum, and D. Vollmer, 2006, Rotational motions in seismology: theory, observations, simulation, in R. Teisseyre, M. Takeo, and E. Majewski, eds., Earthquake source asymmetry, structural media and rotation effects: Springer-Verlag, 391-412.

Crampin, S., 1981, A review of wave motion in anisotropic and cracked elastic-media: Wave Motion, 3, no. 4, 343-391.

Crampin, S., 1984, Effective anisotropic elastic-constants for wave propagation through cracked solids: Geophysical Journal of the Royal Astronomical Society, 76, 135-145.

Crampin, S., E. M. Chesnokov, and R. G. Hipkin, 1984, Seismic anisotropy - the state of the art II: Geophysical Journal of the Royal Astronomical Society, 76, 1-16.

Crampin, S., R. A. Stephen, and R. McGonigle, 1982, The polarization of P-waves in anisotropic media: Geophysical Journal of the Royal Astronomical Society, 68, 477-485.

de la Puente, J., 2008, Seismic wave propagation for complex rheologies: VDM Verlag.

de la Puente, J., M. Käser, M. Dumbser, and H. Igel, 2007, An arbitrary high order discontinuous Galerkin method for elastic waves on unstructured meshes IV: Anisotropy: Geophysical Journal International, 169, no. 3, 1210-1228

Dumbser, M., and M. Käser, 2006, An arbitrary high order discontinuous Galerkin method for elastic waves on unstructured meshes II: the three-dimensional isotropic case: Geophysical Journal International, 167, no. 1, 319-336.

Ferreira, A., and H. Igel, 2009, Rotational motions of seismic surface waves in a laterally heterogeneous earth: Bulletin of the Seismological Society of America, 99, 2B, 1429-1436.

Fichtner, A., and H. Igel, 2009, Sensitivity densities for rotational ground motion measurements: Bulletin of the Seismological Society of America, 99, 2B, 1302-1314.

Guéguen, Y., and J. Sarout, 2009, Crack-induced anisotropy in crustal rocks: predicted dry and fluid-saturated Thomsen's parameters: Physics of the Earth and Planetary Interiors, 172, no. 1-2, 116-124.

Igel, H., A. Cochard, J. Wassermann, A. Flaws, U. Schreiber, A. Velikoseltsev, and N. D. Pham, 2007, Broadband observations of earthquake induced rotational ground motions: Geophysical Journal International, 168, no. 1, 182-196.

Igel, H., U. Schreiber, A. Flaws, B. Schuberth, A. Velikoseltsev, and A. Cochard, 2005, Rotational motions induced by the M8.1 Tokachi-oki earthquake, September 25, 2003: Geophysical Research Letters, 32, L08309.

Larner, K., 1993, Dip-moveout error in transversely isotropic media with linear velocity variation in depth: Geophysics, 58, 1442-1453.

Lee, H. K. W., H. Igel, and M. D. Trifunac, 2009a, Recent advances in rotational seismology: Seismological Research Letters, 80, no. 3, 479-490.

Lee, H. K. W., M. Çelebi, M. I. Todorovska, and H. Igel, 2009b, Introduction to the special issue on rotational seismology and engineering applications: Bulletin of the Seismological Society of America, 99, 2B, 945-957.

McLeod, D. P., G. E. Stedman, T. H. Webb, and U. Schreiber, 1998, Comparison of standard and ring laser rotational seismograms: Bulletin of the Seismological Society of America, 88, 1495-1503.

Musgarve, M. J. P., 1970, Crystal acoustics: Holden-Day.

Nigbor, R. L., J. R. Evans, and C. R. Hutt, 2009, Laboratory and field testing of commercial rotational seismometers: Bulletin of the Seismological Society of America, 99, 2B, 1215-1227. 
Pancha, A., T. H. Webb, G. E. Stedman, D. P. McLeod, and U. Schreiber, 2000, Ring laser detection of rotations from teleseismic waves: Geophysical Research Letters, 27, no. 21, 3553-3556.

Pšenčík, I., and D. Gajewski, 1998, Polarization, phase velocity and NMO velocity of qP-waves in arbitrary weakly anisotropic media: Geophysics, 63, 1754-1766.

Pham, D. N., H. Igel, J. Wassermann, A. Cochard, and U. Schreiber, 2009a The effects of tilt on interferometric rotation sensors: Bulletin of the Seismological Society of America, 99, 2B, 1352-1365

Pham, D. N., H. Igel, J. Wassermann, M. Käser, J. de la Puente, and U. Schreiber, 2009b, Observations and modelling of rotational signals in the P-coda: constraints on crustal scattering: Bulletin of the Seismological Society of America, 99, 2B, 1315-1332.

Sams, M. S., M. H. Worthington, M. S. King, and M. S. Khanshir, 1993, A comparison of laboratory and field measurements of P-wave anisotropy: Geophysical Prospecting, 41, no. 2, 189-206.

Schreiber, U., G. E. Stedman, H. Igel, and A. Flaws, 2006, Ring laser gyroscopes as rotation sensors for seismic wave studies, in $\mathrm{R}$. Teisseyre, $\mathrm{M}$. Takeo, and E. Majewski, eds., Earthquake source asymmetry, structural media and rotation effects: Springer-Verlag, 377-390.

Schreiber, K. U., J. N. Hautmann, A. Velikoseltsev, J. Wassermann, H. Igel J. Otero, F. Vernon, and J. P. R. Wells, 2009, Ring laser measurements of ground rotations for seismology: Bulletin of the Seismological Society of America, 99, 2B, 1190-1198.

Stein, S., and M. Wysession, 2003, An introduction to seismology, earthquake, and earth structure: Blackwell Press.
Suryanto, W., H. Igel, J. Wassermann, A. Cochard, B. Schuberth, D. Vollmer, F. Scherbaum, U. Schreiber, and A. Velikoseltsev, 2006, First comparison of array-derived rotational ground motions with direct ring laser measurements: Bulletin of the Seismological Society of America, 96, no. 6, 2059-2071.

Takeo, M., and H. M. Ito, 1997, What can be learned from rotational motions excited by earthquakes?: Geophysical Journal International, 129, no. 2, 319-329.

Thomsen, L., 1986, Weak elastic anisotropy: Geophysics, 51, 1954-1966.

Tsvankin, I., 1995a, Seismic wavefields in layered isotropic media: Samizdat Press.

Tsvankin, I., 1995b, Body-wave radiation patterns and AVO in transversely isotropic media: Geophysics, 60, 1409-1425.

Tsvankin, I., 1996, P-wave signatures and notation for transversely isotropic media: an overview: Geophysics, 61, 467-483.

Tsvankin, I., and L. Thomsen, 1994, Nonhyperbolic reflection moveout in anisotropic media: Geophysics, 59, 1290-1304.

Wang, Z., 2002, Seismic anisotropy in sedimentary rocks. Part 2: laboratory data: Geophysics, 67, 1423-1440.

Wassermann, J., S. Lehndorfer, H. Igel, and U. Schreiber, 2009, Performance test of a commercial rotational motion sensor: Bulletin of the Seismological Society of America, 99, 2B, 1449-1456.

Zheng, X., and I. Pšenčík, 2002, Local determination of weak anisotropy parameters from $\mathrm{qP}$-wave slowness and particle motion measurements: Pure and Applied Geophysics, 159, no. 7-8, 1881-1905. 